\title{
N-body Simulations to Test the Reliability of Two-point Correlation Functions of Galaxies
}

\author{
YASUSHI SuTO \\ Uji Research Center \\ Yukawa Institute for Theoretical Physics \\ Kyoto University, Uji 611, Japan
}

The shape and amplitude of the galaxy - galaxy correlation functions, $\xi_{g g}(r)$, are among the most widely used measures of the large-scale structure in the universe (Totsuji \& Kihara 1969). The estimates, however, might be seriously affected by the limited size of the sample volume, or equivalently, the limited number of available galaxies. In fact, while the observable universe extends $c / H_{0} \sim 3000 h^{-1} \mathrm{Mpc}$, most observational works to map the distribution of galaxies so far have been mainly applied to samples within $\sim 100 h^{-1} \mathrm{Mpc}$ from us. Thus a CfA redshift survey slice, for example, of $8^{\mathrm{h}}<\alpha<17^{\mathrm{h}}, 26.5^{\circ}<\delta<32.5^{\circ}$, and $c z \lesssim 15000 \mathrm{~km} / \mathrm{sec}$ (de Lapparent et al. 1986,1988 ) represents merely $\sim 2 \times 10^{-5}$ of the total volume of the observable universe. This clearly illustrates the importance of examining possible systematic biases and variations in the estimates of two-point correlation functions from instrinsically limited data. We studied such sample-to-sample variations by analysing subsamples extracted from large $\mathrm{N}$-body simulation data.

For the purpose of constructing realizations as similar as possible to the observed galaxy distribution, we adopted a CDM model with nonvanishing cosmological constant (model LB in Suginohara \& Suto 1991), simply because its correlation function (from the entire simulation data) turned out to match very well the observational estimate of $\xi_{g g}(r)$ by Davis \& Peebles (1983). Our simulation employs $N=64^{3}$ particles, and the Hubble constant, $h$, is 1.0 , the present density parameter, $\Omega$, is 0.2 , and the universe is spatially flat by the residual cosmological constant.

In order to estimate the sample-to-sample variations, we randomly extracted four sets of 36 slices from the simulation data with $(\Delta \theta, \Delta \phi$, depth $)=\left(6^{\circ}, 120^{\circ}\right.$, $\left.50 h^{-1} \mathrm{Mpc}\right),\left(12^{\circ}, 120^{\circ}, 50 h^{-1} \mathrm{Mpc}\right),\left(6^{\circ}, 120^{\circ}, 100 h^{-1} \mathrm{Mpc}\right)$, and $\left(12^{\circ}, 120^{\circ}\right.$, $100 h^{-1} \mathrm{Mpc}$ ). Then two-point correlation functions are computed in real space, $\xi(r)$, and in redshift space, $\xi(s)$. In estimating the latter, we applied the Schechter type selection function to our simulation data to obtain magnitude limited samples with $m_{0}<15.5$. We tried three estimates, $\xi_{11}(s), \xi_{1 \varphi}(s)$, and $\xi_{\varphi \varphi}(s)$ on the basis of the three different weighting schemes (de Lapparent et al. 1988). The mean values 
Table 1: The estimates of the correlation lengths in real space and redshift spaces. All values are quoted in units of $h^{-1} \mathrm{Mpc}$.

\begin{tabular}{ccccc} 
slices & $50 h^{-1} \mathrm{Mpc}\left(6^{\circ}\right)$ & $50 h^{-1} \mathrm{Mpc}\left(12^{\circ}\right)$ & $100 h^{-1} \mathrm{Mpc}\left(6^{\circ}\right)$ & $100 h^{-1} \mathrm{Mpc}\left(12^{\circ}\right)$ \\
\hline$\xi(r)$ & $3.9 \pm 1.8$ & $4.2 \pm 1.7$ & $4.6 \pm 1.7$ & $5.1 \pm 1.3$ \\
$\xi_{11}(s)$ & $3.8 \pm 1.7$ & $5.0 \pm 1.6$ & $5.3 \pm 1.8$ & $5.4 \pm 1.8$ \\
$\xi_{1 \varphi}(s)$ & $4.8 \pm 1.7$ & $5.0 \pm 1.6$ & $5.7 \pm 2.2$ & $5.8 \pm 1.8$ \\
$\xi_{\varphi \varphi}(s)$ & $4.8 \pm 1.8$ & $4.9 \pm 1.5$ & $5.8 \pm 2.1$ & $6.0 \pm 1.4$
\end{tabular}

and standard deviations of correlation lengths where $\xi$ is unity are calculated from the four sets of 36 slices (Table 1 ).

Table 1 shows that the fractional variation of the correlation length amounts to $\sim 0.25$ for samples comparable to the CfA redshift surveys $\left(12^{\circ}, 120^{\circ}, 100 h^{-1} \mathrm{Mpc}\right.$ slices). The variations are translated to a factor of two uncertainty of the amplitude of correlation functions. Furthermore if the typical characteristic scale of the clustering is comparable to, or even more than the size of the subsamples, as in the case of the available redshift surveys, correlation functions of the subsamples turned out to systematically underestimate the amplitude of the true correlation (the correlation lengths of the entire data are $5.5 h^{-1} \mathrm{Mpc}$ and $6.4 h^{-1} \mathrm{Mpc}$ in real and redshift spaces, respectively). The degree of such systematic bias toward weaker correlations is highly dependent on the underlying cosmological models and the size of the sample, and thus cannot be predicted a priori. In the above CDM model, the bias ranges from $28 \%$ for $50 \mathrm{~h}^{-1} \mathrm{Mpc} \times 6^{\circ} \times 120^{\circ}$ slices to $6 \%$ for $100 \mathrm{~h}^{-1} \mathrm{Mpc} \times 12^{\circ} \times 120^{\circ}$ slices. These variations and biases are important sources to be kept in mind in normalizing the theoretical models by correlation functions. The detail of the present analysis will be reported elsewhere (Itoh et al. 1991).

It is my pleasure to thank Makoto Itoh and Tatsushi Suginohara for many interesting discussions and contributions. Travel support from Yukawa Memorial Foundation, and the Kyoto University Foundation is gratefully acknowledged. The numerical simulation were carried out on the Hitac S-820/80 at KEK (National Laboratory for High Energy Physics, Japan).

\section{References}

Davis, M., Peebles, P.J.E., 1983, Ap.J., 267, 465.

de Lapparent, V., Geller, M.J., Huchra, J.P., 1986, Ap.J.(Letters), 302, L1.

de Lapparent, V., Geller, M.J., Huchra, J.P., 1988, Ap.J., 332, 44.

Suginohara, T., Suto, Y., 1991, Publ.Astron.Soc.Japan., 43, L17.

Itoh, M., Suginohara, T., Suto, Y., 1991, Ap.J., submitted.

Totsuji, H., Kihara, T., 1969, Publ.Astron.Soc.Japan., 21, 221. 